\title{
On site monitoring of Grana Padano cheese production using portable spectrometers
}

\author{
Laura Marinoni, ${ }^{\text {a* }}$ Angelo Stroppa, ${ }^{\mathrm{b}}$ Stefania Barzaghi, ${ }^{a}$ Katia Cremonesi, ${ }^{\mathrm{a}}$ Nicolò Pricca, ${ }^{\mathrm{a}}$ Aurora Meucci, ${ }^{a}$ \\ Giulia Maria Pedrolini, ${ }^{\mathrm{C}}$ Andrea Gallia and Giovanni Cabassi ${ }^{\mathrm{a}}$ \\ ${ }^{a}$ CREA-ZA, Council for Agricultural Research and Economics - Research Centre for Animal Production and Aquaculture, Lodi, Italy. \\ *E-mail: laura.marinoni@crea.gov.it

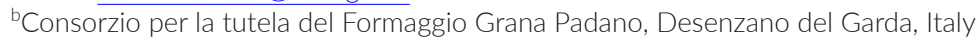 \\ 'Università degli Studi di Milano, Milano, Italy
}

The GRANIR project founded by the Grana Padano Protection Consortium and developed by CREA-ZA research centre is devoted to the development of a rapid and economic method for the chemical characterisation of Grana Padano PDO cheese based on near infrared (NIR) spectroscopy technology. For this purpose, the Consortium purchased several portable spectrometers $\mathrm{XNIR}^{\mathrm{TM}}$ (dinamica generale ${ }^{\circledR}$, Poggio Rusco, MN, Italy), to be assigned to the Consortium staff for screening operations of production batches in the fire-branding step, in warehouses and at the packaging step, on cheese paste. To develop predictive models and to evaluate the performance of the portable instruments, 195 samples of Grana Padano were scanned directly on the whole open wheel, scanning both rind and cheese paste. Robust models were built for the prediction of dry matter, fat, fat/dry matter, proteins and proteins/dry matter content using average spectra of rind and paste and chemical data of cheese paste. Additional spectra acquired with two other instruments were included in order to make the models less sensitive to different instruments. Spectra of the same samples acquired at different temperatures (10, 16 and $25^{\circ} \mathrm{C}$ ) were also added to the dataset in order to reduce the influence of temperature on prediction results. The obtained results showed a satisfactory predictive ability of the models built with portable NIR spectrometers, with respect to the chemical composition of Grana Padano cheese, showing root mean square errors in prediction comparable to that obtained with a Fourier-Transform NIR benchtop instrument. This allows the estimation of average cheese composition, at batch level, using multiple scans taken on a high number of wheels.

\section{Introduction}

Grana Padano is an Italian protected designation of origin (PDO) hard cheese, produced with an artisanal production technology which involves: the use of raw cow milk partially skimmed by natural creaming; the use of a traditional copper vat with a bell shape turned upside down; the use of autochthonous whey starter; a manual curd breaking; a high temperature curd heating; long ripening for at least nine months., ${ }^{1,2}$

The Grana Padano Protection Consortium routinely analyses the cheese composition of 130 associated production dairies. The use of not standardised milk and small differences in the cheesemaking process among the different dairies leads to a final product with heterogeneous chemical, physical and compositional characteristics. Such characteris- tics have to be monitored in order to meet the requirements of the Product Specifications. Classical analytical methods for assessing the quality of cheese are laborious, expensive and require the destruction of the sample. Moreover, the high economic value of the cheese drives to a limited sampling plan.

Thus, because it's a traditional production, the Grana Padano cheesemaking requires high standards of quality and soft and not invasive techniques for process control.

Within the GRANIR collaboration between the CREA-ZA research centre of Lodi and the Grana Padano Protection Consortium, a rapid and economic method was developed for the chemical characterization of Grana Padano PDO

\section{Correspondence}

L. Marinoni (laura.marinoni@crea.gov.it)

doi: $10.1255 /$ nir2017.085

Citation: L. Marinoni, A. Stroppa, S. Barzaghi, K. Cremonesi, N. Pricca,

A. Meucci, G.M. Pedrolini, A. Galli and G. Cabassi, "On site monitoring of

Grana Padano cheese production using portable spectrometers", in Proc. $18^{\text {th }}$

Int. Conf. Near Infrared Spectrosc., Ed by S.B. Engelsen, K.M. Sørensen and F.

van den Berg. IM Publications Open, Chichester, pp. 85-90 (2019). https://

doi.org/10.1255/nir2017.085

\section{(C) 2019 The Authors}

This licence permits you to use, share, copy and redistribute the paper in any medium or any format provided that a full citation to the original paper is given.

ISBN: 978-1-906715-27-4 
cheese based on near infrared (NIR) spectroscopy technology.

For this purpose, the Consortium purchased 12 portable XNIR spectrometers (dinamica generale ${ }^{\circledR}$, Poggio Rusco, MN, Italy) to be assigned to the Consortium staff for screening operations of cheese batches both in warehouses, on whole cheese wheels and at the packaging step, on cheese paste. The first step of the project involved the standardisation of three instruments.

\section{Materials and methods}

195 slices of Grana Padano DOP cheese of 6-13 months of ripening with an average weight of $4 \mathrm{~kg}$ were sampled by the consortium from several dairies located in the Po valley. Samples were scanned with a portable instrument directly on the whole slice. After the removal of the rind (first $6 \mathrm{~mm}$ from the outside), the cheese was ground and scanned with a benchtop Fourier transform (FT)-NIR NIRFlex N-500 (Buchi Italia, Cornaredo, Italy). Reference data for dry matter, fat and proteins content were generated by chemical analyses performed by three laboratories selected by the Consortium.

Scans with the portable instrument were performed in reflection mode, scanning both the rind and the paste in 10 randomly selected spots in the spectral range of 950-1800 nm. FT-NIR spectra were acquired in duplicate in reflection mode (32 scans, resolution of $4 \mathrm{~cm}^{-1}$ ) in the range of $1000-2500 \mathrm{~nm}$ using a $9 \mathrm{~cm}$ diameter cuvette with quartz bottom.

Predictive models for dry matter (dm), fat (F), fat/dry matter (F/dm), proteins (P) and Proteins/Dry matter (P/ $\mathrm{dm})$ were computed by partial least square (PLS) regression using average spectra of rind and paste and chemical data of cheese paste. The models were developed using MATLAB 7.0 (The MathWorks, Inc., USA) and PLS ToolBox 8.0 (Eigenvector, USA).

\section{Results}

In order to reduce the measurement uncertainty, the reference chemical analyses were performed by three laboratories. Through a suitable set of repeated blind samples, it was possible to evaluate the repeatability and reproducibility associated with each analytical parameter. The analysis of chemical data also allowed for the removal of outliers, as identified through the application of the $\mathrm{Q}$ test. ${ }^{3}$

Spectral outliers were identified and removed by $\mathrm{Q}$ residual vs $\mathrm{T} 2$ graphics and by leverage.

Figure 1 shows the average NIR absorbance spectra of cheese rind used in the calibration development, showing the characteristic water absorption band around $1460 \mathrm{~nm}$ of the first overtone of the $\mathrm{OH}$ stretch vibration and the band at $1200 \mathrm{~nm}$, related to the $-\mathrm{CH}$ stretch of the second overtone, which arise from fat component. The strong water absorption region hides the proteinrelated absorption bands, such as the overtone of the $\mathrm{NH}$ bond at $1500 \mathrm{~nm}^{4}{ }^{4}$

Predictive models for cheese composition were built with:

spectra acquired on the paste or on the rind of the 195 samples scanned at $16^{\circ} \mathrm{C}$, which is the common temperature of the warehouses;

spectra of samples scanned at $10^{\circ} \mathrm{C}$ and $25^{\circ} \mathrm{C}$ to include variability among the producers in the management of the warehouses;

The dataset was divided into calibration and validation sets, making the selection through the Kennard-Stone duplex algorithm. ${ }^{5}$

\section{Predictive models built with cheese paste spectra}

Descriptive statistic of calibration and validation datasets used to build predictive models are reported in Table 1.

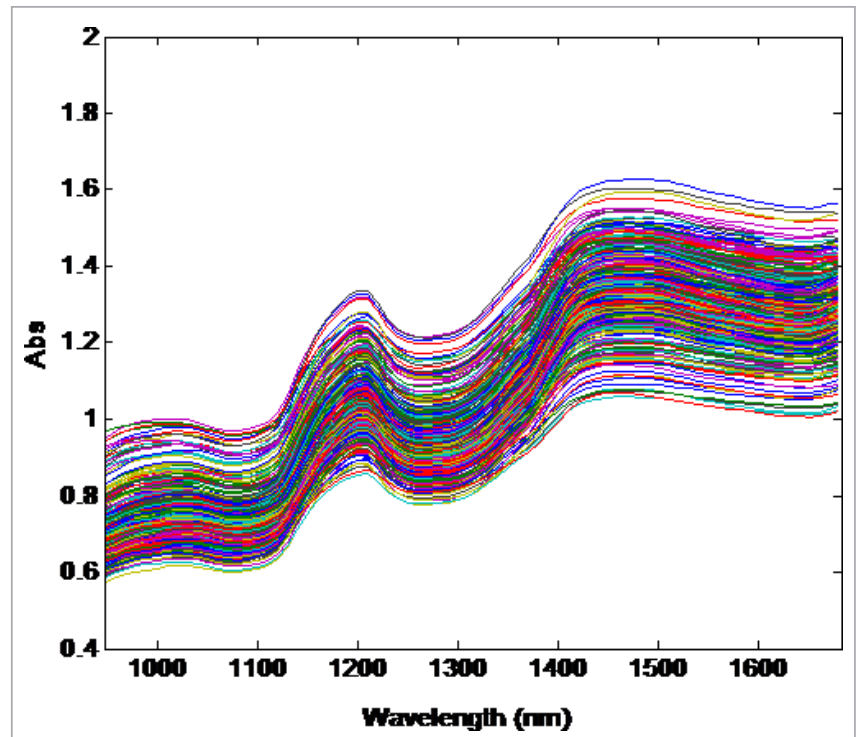

Figure 1. Absorbance spectra of cheese rind. 
Table 1. Descriptive statistics of the calibration and validation datasets used for the predictive PLS models.

\begin{tabular}{|l|c|c|c|c|c|c|c|c|}
\hline & \multicolumn{4}{|c|}{ Calibration $(\boldsymbol{n}=116)$} & \multicolumn{4}{c|}{ Validation $(\boldsymbol{n}=74)$} \\
\hline & $\min$ & $\max$ & mean & Std dev & $\min$ & $\max$ & mean & Std dev \\
\hline $\mathrm{dm}(\%)$ & 64.18 & 70.16 & 67.22 & 1.11 & 65.37 & 69.49 & 67.08 & 1.06 \\
\hline $\mathrm{F}(\%)$ & 23.98 & 32.90 & 28.46 & 1.65 & 24.49 & 32.84 & 28.91 & 1.50 \\
\hline $\mathrm{P}(\%)$ & 29.29 & 36.24 & 32.42 & 1.08 & 30.66 & 34.97 & 32.31 & 0.95 \\
\hline $\mathrm{F} / \mathrm{dm}(\%)$ & 35.85 & 48.71 & 42.32 & 2.19 & 37.17 & 48.42 & 43.08 & 1.94 \\
\hline $\mathrm{P} / \mathrm{dm}(\%)$ & 43.36 & 54.20 & 48.26 & 1.81 & 45.20 & 53.07 & 48.18 & 1.61 \\
\hline
\end{tabular}

Table 2. Characteristics of predictive models built with the paste spectra.

\begin{tabular}{|l|c|c|c|c|c|}
\hline Preprocessing & $\begin{array}{c}\mathrm{F} / \mathrm{dm} \\
1^{\text {st }} \text { Der (order } \\
\text { 2, window 3 pt) } \\
\text { SNV, MC }\end{array}$ & $\begin{array}{c}1^{\text {st }} \text { Der (order 2, } \\
\text { window 3 pt), } \\
\text { SNV, Autoscale }\end{array}$ & $\begin{array}{c}1^{\text {st }} \text { Der (order 2, } \\
\text { window 3 pt), } \\
\text { MSC (mean) }\end{array}$ & $\begin{array}{c}1^{\text {st }} \text { Der (order 2, } \\
\text { window 3 pt), } \\
\text { SNV, MC }\end{array}$ & $\begin{array}{c}2^{\text {nd }} \text { Der (order } \\
\text { 2, window } \\
5 \mathrm{pt} \text { ), MC }\end{array}$ \\
\hline PC & 6 & 7 & 7 & 7 & 7 \\
\hline RMSEC & 0.524 & 0.396 & 0.388 & 0.299 & 0.467 \\
\hline RMSECV & 0.644 & 0.518 & 0.470 & 0.378 & 0.582 \\
\hline RMSEP & 0.602 & 0.580 & 0.461 & 0.396 & 0.654 \\
\hline Bias & $-1.42 \mathrm{E}-14$ & $3.55 \mathrm{E}-14$ & $2.47 \mathrm{E}-08$ & $7.11 \mathrm{E}-15$ & $0.00 \mathrm{E}+00$ \\
\hline CV & -0.006 & 0.003 & -0.015 & 0.003 & -0.005 \\
\hline Pred & -0.023 & -0.016 & -0.021 & 0.034 & -0.004 \\
\hline$R_{\text {Cal }}^{2}$ & 0.919 & 0.933 & 0.917 & 0.904 & 0.746 \\
\hline$R_{\text {BV }}^{2}$ & 0.882 & 0.891 & 0.882 & 0.853 & 0.620 \\
\hline$R_{\text {Pred }}^{2}$ & 0.902 & 0.870 & 0.907 & 0.832 & 0.616 \\
\hline
\end{tabular}

$1^{\text {st }}$ Der: first derivative; SNV: Standard Normal Variate; MC: mean centre; MSC: Multiplicative Scatter Correction; 2nd Der: second derivative; PC: number of principal components; RMSEC: root mean square error of calibration (\%); RMSECV: root mean square error of cross-validation (\%); RMSEP: root mean square error of prediction (\%); CV bias: bias in cross validation; Pred bias: bias in prediction; $\mathrm{R}_{\text {cal }}^{2} \mathrm{R}^{2}$ in calibration; $\mathrm{R}_{\mathrm{CV}}^{2}$ : $R^{2}$ in cross validation; $R_{\text {Pred }}^{2}: R^{2}$ in prediction.

Table 2 summarises the performance of the calibration models developed using the cheese paste spectra.

Best spectral pretreatments were chosen by optimising cross-validation models using the "PLS model optimiser" tool. The best cross-validation models were then tested on an independent test-set. The random subsets (eight splits and five iterations) cross validation was used for selecting the optimal number of principal components. They were chosen based on the minimisation of the cross-validation error while also taking into account the significance of the components as function of the signalto-noise ratio. Models performance were evaluated by considering errors, $R^{2}$, and bias both in cross validation (RMSECV, $R_{\text {val }}^{2}, C_{\text {bias }}$ ) and in prediction (RMSEP, $R_{\text {pred }}^{2}$, Pred. Bias $_{\text {. }}$.
Cross validation errors were fairly low in all cases, ranging from $0.4 \%$ in predicting protein content to $0.6 \%$ in the $\mathrm{F} / \mathrm{dm}$ model. Prediction errors ranged between 0.4 for the P model and $0.65 \%$ for F/dm. The $R_{\text {pred }}^{2}$ are close to or equal to 0.9 , with the exception of the $\mathrm{dm}$ model. Bias were negligible in all cases.

The results of this activity show the good performance of the portable instrument in predicting the chemical composition of cheese with errors in prediction comparable to the analytical error of the reference methods: $\mathrm{F}=0.68 \%, \mathrm{P}=0.38 \%$ and $\mathrm{dm}=0.78 \%$. Prediction errors were also comparable to those obtained with a benchtop FT-NIR working on grinded paste: 0.67, 0.47, 0.54, 0.49 and $0.71 \mathrm{~g} / 100 \mathrm{~g}$ for F/dm, P/dm, F, P and dm respectively. Figure 2 shows the scatter plots of the five models. 

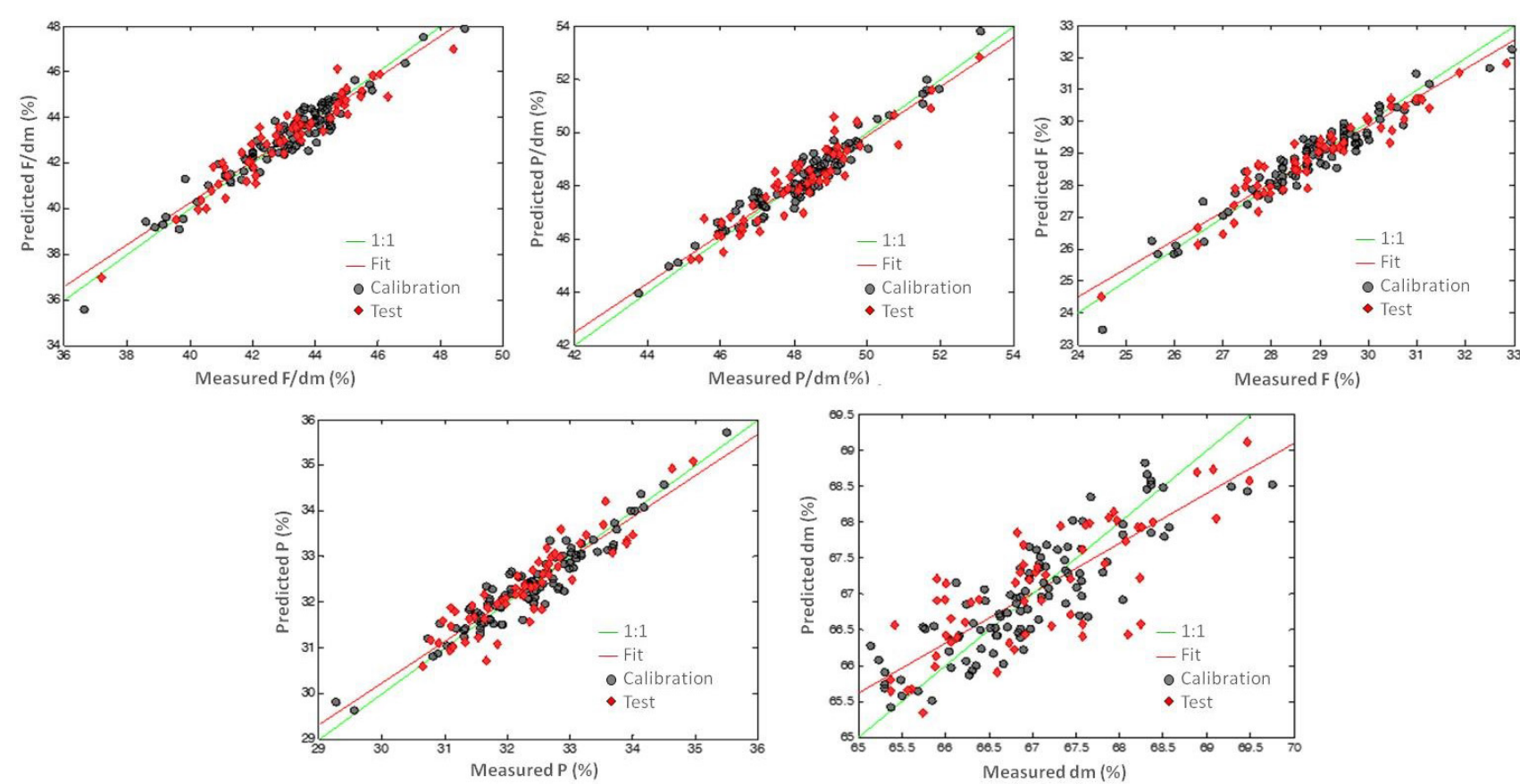

Figure 2. Scatter plot of cross validation and prediction performance of predictive models built with paste spectra (black dots represent the samples used to build the model while the red ones are the samples used in independent validation. The green line is the bisector $1: 1$ ( $y$ is equal to y measured); the red line is the one that best interpolates the data in analysis).

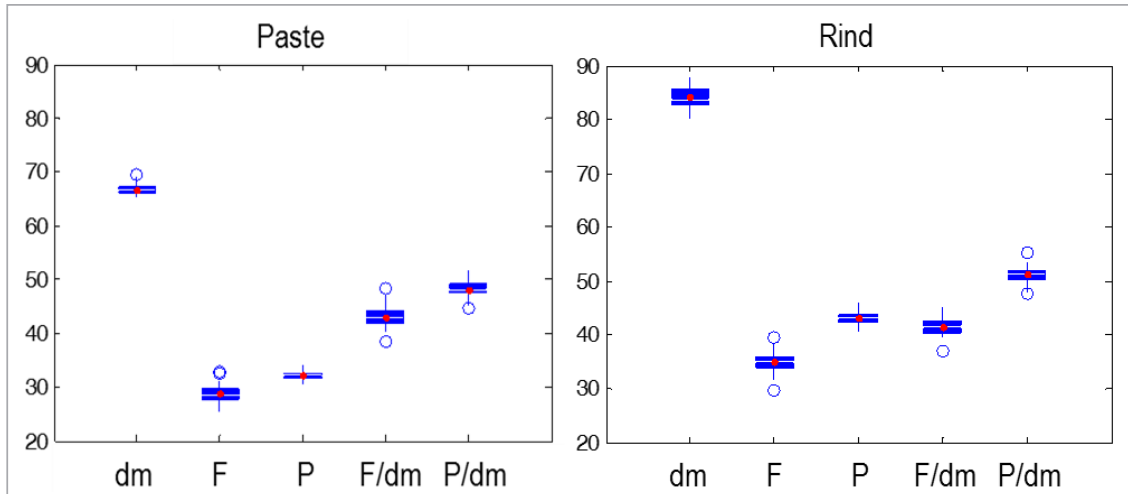

Figure 3. Paste (on the left) and rind (on the right) chemical composition (average reference data of 40 samples, \%). Vertical blue line indicates data range; horizontal blue line indicates interquartile range median; red dots are the mean values; white dots are outliers.

\section{Predictive models built with cheese rind spectra}

The monitoring of cheese composition by scanning the cheese rind scan was more challenging. Even if the composition of the dry matter of cheese paste and rind is similar, the dry matter content is very different (Figure 3). Thus, predictive models were built for the ratio parameters F/dm and $\mathrm{P} / \mathrm{dm}$, which are the most constant parameters and less sensitive to the moisture gradient inside the cheese.
Table 3 summarises the performance of the calibration models developed (basic models). Prediction errors were $1.189 \%$ for the F/dm model and $0.938 \%$ for the $\mathrm{P} / \mathrm{dm}$ model. $\mathrm{R}_{\text {pred }}^{2}$ were close to 0.6 .

In order to obtain more robust models, additional spectra $(n=20)$ acquired at different temperatures were included in the calibration set, in order to make models less sensitive to the different warehouses' management. Results are showed in Table 3 (extended 
Table 3. Characteristics of predictive models built with the rind spectra.

\begin{tabular}{|c|c|c|c|c|}
\hline & \multicolumn{2}{|c|}{ Basic models $(n=190)$} & \multicolumn{2}{|c|}{ Extended models $(n=209)$} \\
\hline & $\mathrm{F} / \mathrm{dm}$ & $\mathrm{P} / \mathrm{dm}$ & $\mathrm{F} / \mathrm{dm}$ & $\mathrm{P} / \mathrm{dm}$ \\
\hline Preprocessing & $\begin{array}{l}1^{\text {st }} \text { Der (order 2, } \\
\text { window } 5 \mathrm{pt} \text {, } \\
\text { MSC (mean), MC }\end{array}$ & $\begin{array}{l}1^{\text {st }} \text { Der (order } 2, \\
\text { window } 3 \mathrm{pt} \text { ), } \\
\text { Pareto (Sqrt Std) } \\
\text { Scaling }\end{array}$ & $\begin{array}{c}1^{\text {st }} \text { Der (order 2, } \\
\text { window } 5 \text { pt), MSC } \\
\text { (mean), MC }\end{array}$ & $\begin{array}{l}1^{\text {st }} \text { Der (order } 2, \\
\text { window } 3 \text { pt), Pareto } \\
\text { (Sqrt Std) Scaling }\end{array}$ \\
\hline PC & 7 & 6 & 8 & 7 \\
\hline RMSEC & 0.996 & 0.821 & 1.024 & 0.868 \\
\hline RMSECV & 1.191 & 0.956 & 1.159 & 0.952 \\
\hline RMSEP & 1.189 & 0.938 & 1.177 & 0.884 \\
\hline Bias & $-2.13 \mathrm{E}-14$ & 0.006796 & $0.00 \mathrm{E}+00$ & 0.000 \\
\hline $\mathrm{CV}_{\text {Bias }}$ & 0.000 & 0.023 & 0.004 & -0.002 \\
\hline Pred $_{\text {Bias }}$ & -0.083 & 0.011 & -0.123 & -0.018 \\
\hline $\mathrm{R}_{\text {Cal }}^{2}$ & 0.731 & 0.727 & 0.744 & 0.717 \\
\hline$R_{C V}^{2}$ & 0.633 & 0.641 & 0.678 & 0.664 \\
\hline $\mathrm{R}_{\text {Pred }}^{2}$ & 0.588 & 0.593 & 0.595 & 0.655 \\
\hline
\end{tabular}

$1^{\text {st }}$ Der: first derivative; MSC: Multiplicative Scatter Correction; MC: mean centre; PC: number of principal components; RMSEC: root mean square error of calibration (\%); RMSECV: root mean square error of cross-validation (\%); RMSEP: root mean square error of prediction (\%); CV bias: bias in cross validation; Pred bias: bias in prediction; $R_{c a l}^{2}: R^{2}$ in calibration; $R_{C V}^{2}: R^{2}$ in cross validation; $R_{\text {Pred }}^{2}: R^{2}$ in prediction.

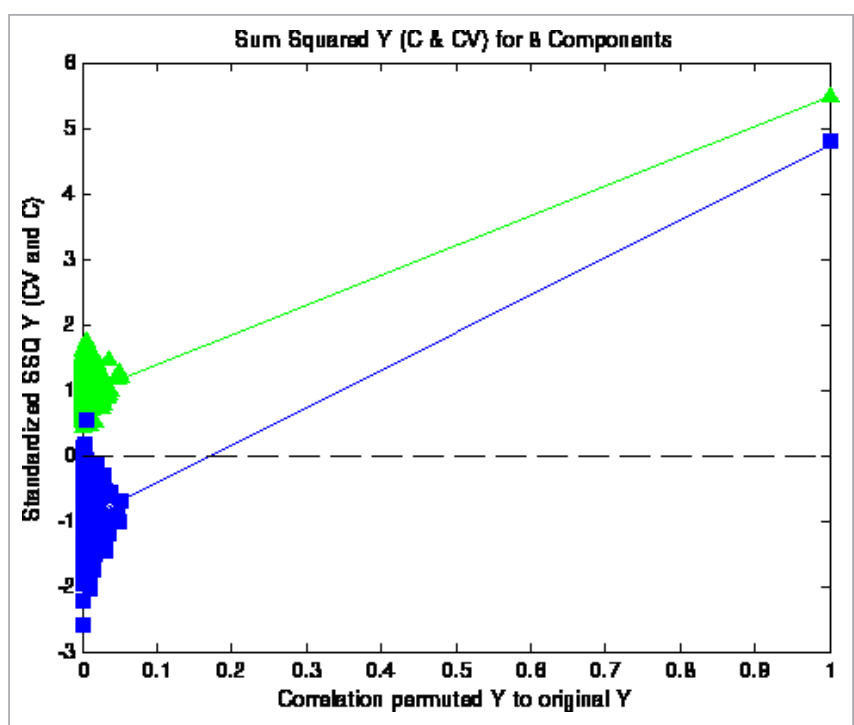

Figure 4. Results of permutation test performed on F/dm basic model.

\section{Conclusions}

The results of this activity showed a good predictive ability of the models built with portable NIR spectrometers, with respect to the chemical composition of Grana Padano cheese. This allows the use of portable NIR spectrometer both for quality control at the packaging step, with the preservation of the sample, and the estimation of the average cheese composition, at batch level.

The use of multiple scans taken on a high number of wheels reduces the sampling error due to sample averaging, even with a RMSEP higher on the single wheel. The possibility to work on a wide sampling base reduce the risk of low representativeness.

\section{References}

1. EU, Approving non-minor amendments to the specification for a name entered in the register of protected designations of origin and protected geographical indications (Grana Padano (PDO)). Commission implementing regulation, EU, No in comparison to paste models, but they were statis tical significant at the permutation test ( $n$ permutation = 1000; Figure 4). 
584/2011, Brussels, Belgium. Official Journal of the European Union, L 160, pp. 65-70 (2011).

2. Grana Padano protection consortium specification rules for the production of Grana Padano (2017). https://www.granapadano.it/en-uk/productionspecification-rules.aspx

3. D.A. Skoog, D.M. West, F.J. Holler and S.R. Crouch, Fundamentals of Analytical Chemistry. Cengage Learning, Belmont, USA (2013).

4. J. Workman, Jr and L. Weyer, Practical Guide to Interpretive Near-Infrared Spectroscopy. CRC Press, Boca Raton, Florida (2007).

5. R.D. Snee "Validation of regression models: methods and examples", Technometrics 19, 415-428 (1977).

https://doi.org/10.1080/00401706.1977.10489581 